\section{Non-Invasive Imaging in Pulmonary \\ Hypertension - Comprehensive \\ Assessment Using Dual-Layer \\ Spectral Computed Tomography -}

Seitaro Oda, MD, PhD; Masafumi Kidoh, MD, PhD

Yasunori Nagayama, MD, PhD;

Takeshi Nakaura, MD, PhD;

Kyoko Hirakawa, MD, PhD;

Eiichiro Yamamoto, MD, PhD;

Kenichi Tsujita, MD, PhD; Toshinori Hirai, MD, PhD

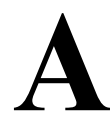

33-year-old woman with pulmonary hypertension underwent computed tomography (CT) imaging using a dual-layer spectral detector CT (IQon Spectral CT; Philips Healthcare, Best; The Netherlands). A comprehensive assessment scan protocol (Supplementary Table) was used. During early (1 $\left.1^{\text {st }}\right)$ phase imaging, highresolution pulmonary CT angiography revealed multiple chronic thrombus in the bilateral distal pulmonary arteries (Figure A). Pulmonary perfusion imaging revealed the functional consequences by delineating multiple segmental perfusion defects in the areas corresponding to the thrombus (Figure B, Supplementary Movie, Supplementary Figure). Delayed $\left(2^{\text {nd }}\right)$ phase cardiac CT revealed right ventricular insertion point late iodine enhancement (LIE), which is indicative of focal myocardial fibrosis (Figure C). In addition, myocardial extracellular volume (ECV) fraction in the affected segments was elevated to 35-48\% (normal range, 23-28\%), suggesting severe right ventricular dys- function (Figure D). The patient was eventually diagnosed with chronic thromboembolic pulmonary hypertension.

Dual-layer spectral detector CT enables comprehensive evaluation of these patients using high-resolution pulmonary $\mathrm{CT}$ angiography and pulmonary perfusion imaging with myocardial LIE imaging, and ECV analysis. ${ }^{1} \mathrm{We}$ believe that this technology offers a practical and useful approach for the non-invasive "one-stop shop" evaluation of pulmonary hypertension.

\section{Disclosures}

K.T. is a member of Circulation Journal's Editorial Team.

\section{Reference}

1. Oda S, Emoto T, Nakaura T, Kidoh M, Utsunomiya D, Funama $\mathrm{Y}$, et al. Myocardial late iodine enhancement and extracellular volume quantification with dual-layer spectral detector dual-energy cardiac CT. Radiol Cardiothorac Imaging 2019; 1: ryct.2019180003.

\section{Supplementary Files}

Supplementary Movie. Pulmonary perfusion imaging by CT.

Please find supplementary file(s);

http://dx.doi.org/10.1253/circj.CJ-20-1007

Received September 30, 2020; revised manuscript received November 28, 2020; accepted December 7, 2020; J-STAGE Advance Publication released online January 30, 2021 Time for primary review: 11 days

Department of Diagnostic Radiology (S.O., M.K., Y.N., T.N., T.H.), Department of Cardiovascular Medicine (K.H., E.Y., K.T.), Faculty of Life Sciences, Kumamoto University, Kumamoto, Japan

Mailing address: Seitaro Oda, MD, PhD, Department of Diagnostic Radiology, Faculty of Life Sciences, Kumamoto University, 1-1-1 Honjo, Chuo-ku, Kumamoto 860-8556, Japan. E-mail: seisei0430@nifty.com

All rights are reserved to the Japanese Circulation Society. For permissions, please e-mail: cj@j-circ.or.jp

ISSN-1346-9843
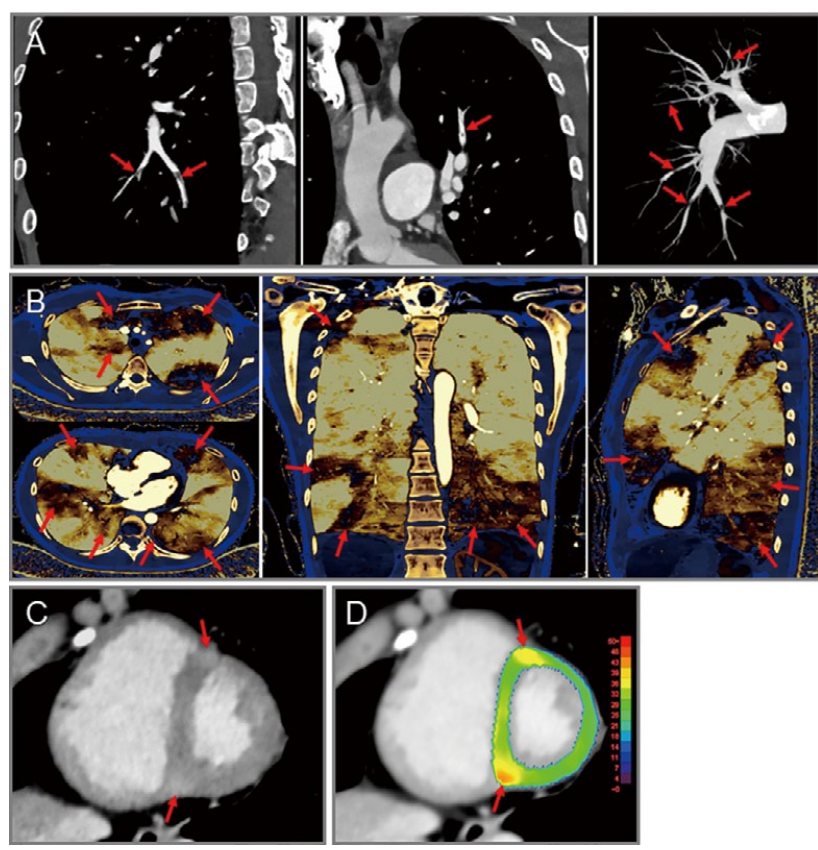

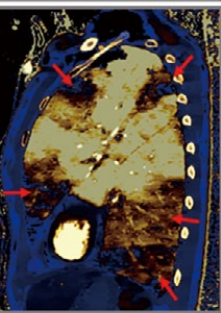

\%

Figure. (A) High-resolution pulmonary computed tomography (CT) angiography revealed multiple chronic thrombus in the pulmonary arteries. (B) Pulmonary perfusion imaging revealed functional consequences by delineating multiple segmental perfusion defects in the areas corresponding to the thrombus. (C) Delayed phase cardiac CT revealed right ventricular insertion point late iodine enhancement. (D) Myocardial extracellular volume fraction in the affected segments was elevated to $35-48 \%$. 